CLINICAL STUDY

\title{
Quality of life is decreased in female patients treated for microprolactinoma
}

\author{
M Kars, A A van der Klaauw, C S Onstein, A M Pereira and J A Romijn \\ Department of Endocrinology and Metabolic Diseases, C4-R, Leiden University Medical Center, PO Box 9600, 2300 RC Leiden, The Netherlands \\ (Correspondence should be addressed to M Kars; Email: m.kars@lumc.nl)
}

\begin{abstract}
Objective: Most studies on treatment of microprolactinoma have focused on clinical and biochemical outcome rather than on functional and mental well-being. We evaluated this topic in female patients with microprolactinoma, because other pituitary adenomas are associated with decreased quality of life. Design: We conducted a cross-sectional study.

Patients and methods: To assess the impact of treatment for microprolactinoma on subjective well-being, quality of life was investigated in 55 female patients (mean age $45 \pm 10$ years), treated for microprolactinoma in our center, using four validated, health-related questionnaires: Short-Form-36 (SF-36), Nottingham Health Profile (NHP), Multidimensional Fatigue Inventory (MFI-20), and Hospital Anxiety and Depression Scale (HADS). Patient outcomes were compared with those of 183 female controls with equal age distributions.

Results: Anxiety and depression scores were increased when compared with controls for all subscales as measured by HADS, and fatigue for all but one subscale as measured by MFI-20. Patients treated for microprolactinoma had worse scores on social functioning, role limitations due to physical problems (SF-36), energy, emotional reaction, and social isolation (NHP) when compared with control subjects. Important independent predictors of quality of life were reproductive status and anxiety and depression scores according to the HADS.

Conclusion: Quality of life is impaired in female patients treated for microprolactinoma, especially due to increased anxiety and depression. These increased anxious and depressive feelings might be due to possible effects of hyperprolactinemia on the central nervous system. Failure to recognize this association may adversely affect patient-doctor relationships.
\end{abstract}

European Journal of Endocrinology 157 133-139

\section{Introduction}

Prolactinomas, especially microprolactinomas, are the most prevalent pituitary adenomas (1-3). Dopamine agonist therapy is the treatment of choice for microprolactinoma (4). Up to $70 \%$ of patients with microprolactinoma treated with dopamine agonist therapy for several years may achieve long-term remission, evidenced by normoprolactinemia, following drug withdrawal $(5,6)$.

Most studies on treatment of microprolactinoma have focused on biochemical outcome rather than functional recovery and well-being. However, other pituitary adenomas are associated with decreased quality of life (7). Quality of life is decreased in patients previously treated for acromegaly, Cushing's disease, and nonfunctioning pituitary macroadenomas (8-14). Moreover, exposure to excessive endogenous growth hormone or glucocorticoid concentrations in acromegaly and Cushing's disease respectively can cause irreversible signs and symptoms, which persist despite long-term cure of the disease $(8,9)$. In patients previously treated for nonfunctioning pituitary macroadenomas, the presence of multiple pituitary deficiencies is a predominant predictor of decreased quality of life (10). Despite mimicry of endocrine homeostasis by optimal hormone replacement strategies, persistent imperfections in endocrine replacement therapies most likely result in subtle physiological derangements and impaired quality of life (15).

Evaluation of quality of life parameters in microprolactinoma will give more insight into the cause of the impaired quality of life in pituitary adenomas. Many factors that influence quality of life in other pituitary adenomas are absent in microprolactinomas, including mass effects of pituitary macroadenoma, hypopituitarism, the effects of surgery, and/or radiotherapy. However, most studies on quality of life in pituitary disease were not focused on prolactinoma. To our knowledge, only one study has been performed in prolactinoma patients. Johnson et al. found impairment in mental health measures in 39 patients with macro- or microprolactinomas when compared with a normal population, assessed by the Short-Form-36 (SF-36) questionnaire (7). Therefore, the purpose of the present study was to evaluate a 
broad spectrum of physical, psychological, and social health aspects of quality of life in female patients treated for microprolactinoma. We assessed quality of life in female patients with microprolactinoma treated, previously or currently, in our center with dopamine agonists, using four validated, health-related quality of life questionnaires (SF-36, Nottingham Health Profile (NHP), Multidimensional Fatigue Inventory (MFI-20), and Hospital Anxiety and Depression Scale (HADS)). We compared the results with those of a control group with equal sex and age distributions (Fig. 1).

\section{Patients and methods}

\section{Protocol}

To assess quality of life in patients treated for prolactinoma, we conducted a cross-sectional survey of all female patients treated with dopamine agonists for microprolactinoma in our department from 1980 to 2006. Diagnostic criteria for microprolactinoma were serum prolactin levels above $60 \mu \mathrm{g} / \mathrm{l}$ (two times upper border of normal value) and evidence of a pituitary tumor smaller than $10 \mathrm{~mm}$ in diameter on magnetic resonance imaging. Surgically treated patients and patients using drugs known to increase prolactin levels were excluded. Dopamine agonist therapy was started with $2.5 \mathrm{mg} /$ day for bromocriptine, $0.5 \mathrm{mg} /$ week for cabergoline, and $0.075 \mathrm{mg} /$ day for quinagolide, and dose titration was based on clinical response and serum prolactin levels within the reference range. At the moment of assessment, all patients were visiting the outpatient clinic twice yearly. A total of 81 patients were asked to participate, and questionnaires were sent to their homes in prepaid envelopes. After 6 weeks, the patients who had not responded were sent a reminder letter and, thereafter, were contacted by telephone to encourage completion and return of the questionnaires. Each patient was also asked to provide a control person of comparable age and same sex (a relative, friend, or neighbor) to compose a control population with a comparable socio-economic status derived from the same geographical area. The control group was extended by controls derived from other studies in our center, who had been similarly approached $(8-10,16,17)$.

In this study, gonadal axis was assessed by basal hormone measurements. In premenopausal women, hypogonadism was diagnosed by oligomenorrhea or amenorrhea and low gonadotropin levels. Other pituitary hormone levels were screened once yearly, none of the patients showed any pituitary hormone deficiencies, including growth hormone deficiency.

The study protocol was approved by the medical ethics committee of Leiden University Medical Center, and all subjects returning completed questionnaires gave written informed consent for participation in the study.

\section{Questionnaires}

SF-36 The SF-36 questionnaire comprises 36 items and records general well-being during the previous 30 days $(18,19)$. The items are formulated as statements or questions to assess eight health concepts: 1) limitations in physical activities because of health problems, 2) limitations in social activities because of physical or emotional problems, 3) limitations in usual role activities because of physical health problems, 4) bodily pain, 5) general mental health (psychological distress and wellbeing), 6) limitations in usual role activities because of
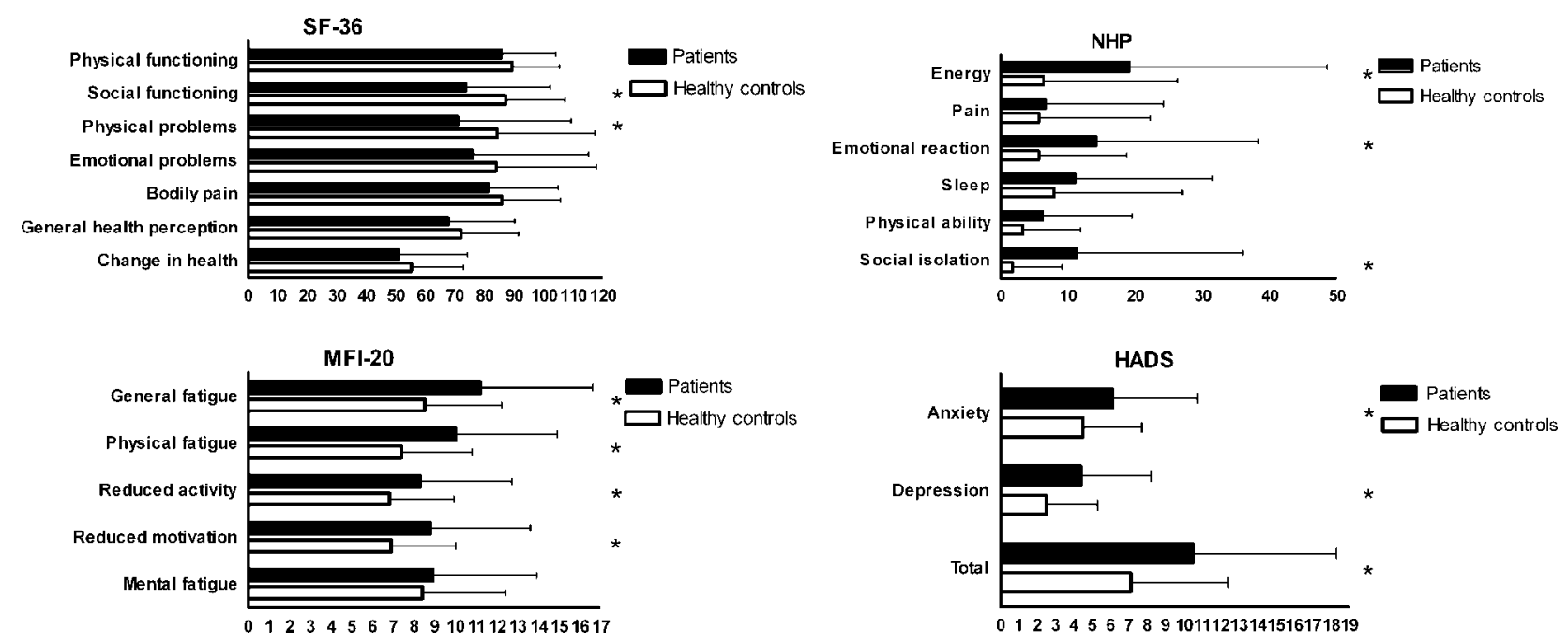

Figure 1 Quality of life in female patients treated for microprolactinoma ( $n=55$, black bars) and healthy controls with the same age and sex distribution ( $n=183$, white bars), according to SF-36, NHP, MFI-20, and HADS. Comparisons showing significant differences between patients and controls are shown by asterisks: ${ }^{*} P<0.05$ patients versus controls. 
emotional problems, 7) vitality (energy and fatigue), and 8 ) general health perceptions and change in health. Since the HADS and the MFI-20 (see below) are more specific questionnaires for mental health, the vitality and general mental health items were left out in this evaluation. Since the scores for the eight items are calculated separately from exclusive item-specific questions (20), the results of the SF-36 items presented in this study are not influenced by the two items left out in this evaluation. Scores are expressed on a 0-100 scale, and higher scores are associated with a better quality of life.

NHP The NHP is frequently used in patients with pituitary disease to assess general well-being and consists of 38 yes/no questions, which are subdivided in six scales assessing impairments, i.e. pain (eight items), energy level (three items), sleep (five items), emotional reactions (nine items), social isolation (five items), and disability/functioning, i.e. physical mobility (eight items) $(21,22)$. Subscale scores are calculated as a weight mean of the associated items and are expressed as a value between 0 and 100. The total score is the mean of the six subscales. A higher score is associated with a worse quality of life.

MFI-20 The MFI-20 comprises 20 statements to assess fatigue, which are measured on a five-point scale (23). Five different dimensions of fatigue (four items each) are calculated from these statements: 1) general fatigue, 2) physical fatigue, 3) reduced activity, 4) reduced motivation, and 5) mental fatigue. Scores vary from 0 to 20 . Higher scores indicate greater experienced fatigue.

HADS The HADS consists of 14 items pertaining to anxiety and depression, which are measured on a fourpoint scale. Scores for the anxiety and depression subscale range from 0 to 21 , and values for the total score range from 0 to 42 . Higher scores indicate more severe anxiety or depression (24). A total score of 13 or more was considered increased.

\section{Assays and normal values}

Baseline prolactin levels were measured using an immunofluorometric assay using WHO-IRP 84/500, with an inter-assay variation coefficient of 3.4-6.2\%, and detection limit of $0.04 \mu \mathrm{g} / \mathrm{l}$ (Wallac Oy, Turku, Finland). At the moment of assessment, prolactin was measured using an electrochemiluminescence immunoassay using Roche Elecsys 1010/2010 and Modular Analytics E170 (Elecsys module, Roche). The interassay variation coefficient was $2.3-3.1 \%$, and the detection limit was $0.47 \mu \mathrm{g} / \mathrm{l}$ (Roche). Normal values for random prolactin levels were $<30 \mu \mathrm{g} / \mathrm{l}$ in women for both assays.

\section{Statistical analysis}

SPSS for Windows version 12.0 (SPSS Inc., Chicago, IL, USA) was used to perform data analysis. Data were expressed as the mean \pm s.D. unless otherwise mentioned. We used the independent samples $t$-test to compare quality of life outcomes between patients and controls. Independent variables affecting quality of life were explored with stepwise linear regression analysis. Differences were considered statistically significant at $P<0.05$.

\section{Results}

\section{Patients and controls}

A total of 62 of the 81 patients $(77 \%)$ responded to our survey. Seven of these patients preferred not to participate. Thus, 55 completed questionnaires $(68 \%)$ were received.

The patient group consisted of 55 female patients, with a mean age of $45 \pm 9.6$ years. Patient characteristics are detailed in Table 1. There were no differences in clinical characteristics between the patients who returned and those who did not return the questionnaires.

Thirty-seven female controls were provided by the 55 patients who returned completed questionnaires. We extended these controls with 146 control persons of comparable age and same sex derived from other studies in our center, who had been similarly approached. Age was equally distributed among patients and controls, $45 \pm 9.6$ vs $46 \pm 9.7$ years.

Table 1 Characteristics of 55 female patients treated for microprolactinoma.

\begin{tabular}{lc}
\hline & Patients $(n=55)$ \\
\hline Age (years) & $45 \pm 9.6$ \\
Pretreatment PRL level $(\mu \mathrm{g} / \mathrm{l})$ & $108.9 \pm 120.8$ \\
Duration of disease $(\mathrm{yr})$ & $14.4 \pm 7.9$ \\
Duration of DA use $(\mathrm{yr})$ & $9.3 \pm 6.9$ \\
Present DA use $(n=27)$ & $11.8 \pm 6.8$ \\
Previous DA use $(n=28)$ & $6.8 \pm 6.2$ \\
Present DA use & $27(49 \%)$ \\
Cabergoline & 16 \\
Quinagolide & 9 \\
Bromocriptine & 2 \\
Present PRL level $(\mu \mathrm{g} / \mathrm{l})$ & $31.9 \pm 44.7$ \\
Present DA use & $19.8 \pm 19.8$ \\
Previous DA use & $40.6 \pm 35.8$ \\
Normoprolactinemia & $67 \%$ \\
Present DA use & $76 \%$ \\
Previous DA use & $53 \%$ \\
Reproductive status & \\
Premenopausal, regular menstrual cycle & $19(35 \%)$ \\
Premenopausal, oral anticonceptives & $5(9 \%)$ \\
Premenopausal, amenorrhea & $14(25 \%)$ \\
Postmenopausal & $17(31 \%)$ \\
\hline
\end{tabular}

Data are given as mean \pm s.D.; DA, dopamine agonist; PRL, prolactin. 


\section{Clinical characteristics}

All 55 patients had been treated with dopamine agonist therapy (bromocriptine, cabergoline, or quinagolide) for microprolactinoma. At the time of completion of the questionnaires, the mean disease duration was 14.4 years, $49 \%(n=27)$ of all the patients were still using dopamine agonists, and $76 \%$ of these patients had a normal prolactin value. Clinical remission, defined as normal prolactin value after withdrawal of dopamine agonists, was present in 53\%. Sixty-nine percent $(n=38)$ of all the women were premenopausal. Of these premenopausal women, 24 (63\%) had regular menses at the time of completion of the questionnaires. Of the 55 patients, 18 had sought treatment for any psychiatric disorder (mainly depression) in the past. Three patients were still receiving medication for their psychiatric disorder; one patient used paroxetine, one fluoxetine, and one patient clozapine.

\section{Quality of life in female patients with micro- prolactinoma when compared with controls}

Patients with microprolactinoma reported impaired quality of life when compared with the 183 control subjects (Table 2). The quality of life scores were significantly reduced in 12 out of 21 subscales when compared with controls.

In patients treated for microprolactinoma, the subscale for social functioning and role limitations due to physical problems of the SF-36, the energy, emotional reaction, and social isolation subscales of the NHP, and the general fatigue, physical fatigue, reduced activity, and reduced motivation subscales of the MFI-20 were impaired when compared with controls. Moreover, the patients also performed worse with respect to anxiety, depression, and total scores for the HADS when compared with controls.

\section{Factors affecting quality of life in female patients with microprolactinoma}

No significant correlations were found between age and scores on the questionnaires in patients or in controls. The present use of dopamine agonists did not influence the scores on the questionnaires. There were no significant correlations between current prolactin concentrations and scores on questionnaires. There were no significant differences on scores of the questionnaires between patients with or without current hyperprolactinemia or between patients with current hyperprolactinemia with or without present use of dopamine agonists. Analysis of premenopausal patients with or without hypogonadism revealed no significant differences on quality of life questionnaires scores.

\section{Linear regression analysis}

Linear regression analysis was performed in a model including age, present use of dopamine agonists, duration of dopamine agonist use, and reproductive status as independent variables and the questionnaire parameters as dependent variables to study factors determining

Table 2 Summary of quality of life assessments in patients and controls.

\begin{tabular}{|c|c|c|c|}
\hline Questionnaire & Patients $(n=55)$ & Controls $(n=183)$ & $P$ value \\
\hline \multicolumn{4}{|l|}{ SF-36 } \\
\hline Physical functioning & $85.5 \pm 18.4$ & $89.2 \pm 16.0$ & NS \\
\hline Social functioning & $73.4 \pm 28.6$ & $87.0 \pm 20.2$ & 0.002 \\
\hline Role limitations due to physical problems & $70.9 \pm 38.1$ & $74.3 \pm 32.9$ & 0.021 \\
\hline Role limitations due to emotional problems & $75.8 \pm 39.3$ & $83.8 \pm 33.9$ & NS \\
\hline Bodily pain & $81.2 \pm 23.6$ & $85.8 \pm 19.6$ & NS \\
\hline General health perception & $67.6 \pm 22.5$ & $72.0 \pm 19.4$ & NS \\
\hline Change in health & $50.9 \pm 23.3$ & $55.2 \pm 17.6$ & NS \\
\hline \multicolumn{4}{|l|}{ NHP } \\
\hline Energy & $19.1 \pm 29.5$ & $6.3 \pm 20.0$ & 0.005 \\
\hline Pain & $6.6 \pm 17.6$ & $5.6 \pm 16.6$ & NS \\
\hline Emotional reaction & $14.2 \pm 24.0$ & $5.6 \pm 13.1$ & 0.017 \\
\hline Sleep & $11.0 \pm 20.4$ & $7.9 \pm 19.0$ & NS \\
\hline Physical ability & $6.2 \pm 13.3$ & $3.4 \pm 8.6$ & NS \\
\hline Social isolation & $11.3 \pm 24.6$ & $1.8 \pm 7.4$ & 0.007 \\
\hline \multicolumn{4}{|l|}{ MFI-20 } \\
\hline General fatigue & $11.2 \pm 5.4$ & $8.5 \pm 3.7$ & 0.001 \\
\hline Physical fatigue & $10.0 \pm 4.9$ & $7.4 \pm 3.4$ & $<0.001$ \\
\hline Reduced activity & $8.3 \pm 4.4$ & $6.8 \pm 3.1$ & 0.020 \\
\hline Reduced motivation & $8.8 \pm 4.8$ & $6.9 \pm 3.1$ & 0.006 \\
\hline Mental fatigue & $8.9 \pm 5.0$ & $8.4 \pm 4.0$ & NS \\
\hline \multicolumn{4}{|l|}{ HADS } \\
\hline Anxiety & $6.1 \pm 4.6$ & $4.5 \pm 3.2$ & 0.022 \\
\hline Depression & $4.4 \pm 3.8$ & $2.5 \pm 2.8$ & 0.001 \\
\hline Total & $10.5 \pm 7.8$ & $7.1 \pm 5.3$ & 0.003 \\
\hline
\end{tabular}

Data are given as mean \pm S.D.; NS, not significant. Patients compared with controls by the independent samples $t$-test. 
quality of life in female patients with microprolactinoma. The increased scores on anxiety, depression, and total anxiety and depression scales according to the HADS, in female patients treated for microprolactinoma when compared with controls, could have influenced the other scores of the quality of life questionnaires. To assess this influence, the total anxiety/depression score of the HADS was also included in the linear regression model as independent variable and the other questionnaire parameters as dependent variables.

Duration of dopamine agonist use and age did not affect any of the scores in the regression model. Present use of dopamine agonist influenced the reduced activity scale of MFI-20. As shown in Table 3, anxiety and depression scores according to the HADS was a significant independent predictor of the other quality of life scales, independently of the other variables. Reproductive status also significantly influenced the scores of general fatigue, physical fatigue, reduced activity, reduced motivation scale (MFI-20), and social functioning scale (SF-36).

\section{Discussion}

The results of the present study demonstrate that multiple aspects of quality of life are impaired in female patients with microprolactinoma after several years of treatment with dopamine agonists when compared with control subjects. These patients perceived reduced well-being, especially due to reduced motivation, fatigue, reduced emotional reaction, and more anxiety and depression. Current use of dopamine agonists appear to influence the results of quality of life on reduced activity, while the present prolactin levels did not affect the results at all. The data indicate subtle, persistent limitations in energy, and psychological and social functioning in patients, currently or previously, treated for microprolactinoma.

Most studies on treatment outcomes of prolactinoma have focused on clinical, biochemical, and tumor volume response rather than on functional and emotional wellbeing. To our knowledge, only Johnson et al. reported a systematic conducted study concerning quality of life in untreated patients with macro- or microprolactinomas, using the SF-36 questionnaire (7). In accordance with our results, their study showed reduced scores in role limitations due to physical and emotional problems, social functioning, vitality, and mental health when compared with scores of the normal population. The present study, however, is the first cross-sectional study in microprolactinoma patients, evaluating various physical and psychological aspects after several years of treatment with dopamine agonists.

It may be argued that selection bias of patients and control subjects may have affected our data. Completed questionnaires were received from $68 \%$ of the patients. However, it seems unlikely that the non-responders have influenced the outcome of our data, because there were no differences in clinical characteristics between responders and non-responders.

This study indicates that patients, previously or currently, treated for microprolactinoma have decreased quality of life in line with results obtained in our center in

Table 3 Linear regression analysis of factors determining quality of life in 55 female patients treated for microprolactinoma.

\begin{tabular}{|c|c|c|c|c|c|}
\hline Questionnaire & Age $(y r)$ & $\begin{array}{c}\text { Present DA } \\
\text { use }(N / Y)\end{array}$ & $\begin{array}{l}\text { Duration of } \\
\text { DA use (yr) }\end{array}$ & $\begin{array}{l}\text { Reproductive } \\
\text { status }\end{array}$ & HADS total score \\
\hline \multicolumn{6}{|l|}{ SF-36 } \\
\hline Physical functioning & & & & & $-0.951(0.004)$ \\
\hline Social functioning & & & & $11.926(0.014)$ & $-2.226(<0.001)$ \\
\hline Role limitations due to physical problems & & & & & $-2.375(<0.001)$ \\
\hline Role limitations due to emotional problems & & & & & $-3.396(<0.001)$ \\
\hline Bodily pain & & & & & $-1.149(0.007)$ \\
\hline General health perception & & & & & $-1.675(<0.001)$ \\
\hline Change in health & & & & & \\
\hline \multicolumn{6}{|l|}{ NHP } \\
\hline Energy & & & & & $2.210(<0.001)$ \\
\hline \multicolumn{6}{|l|}{ Pain } \\
\hline Emotional reaction & & & & & $2.384(<0.001)$ \\
\hline \multicolumn{6}{|l|}{ Sleep } \\
\hline Physical ability & & & & & $0.563(<0.022)$ \\
\hline Social isolation & & & & & $2.195(<0.001)$ \\
\hline \multicolumn{6}{|l|}{ MFI-20 } \\
\hline General fatigue & & & & $-2.624(0.055)$ & $0.409(0.050)$ \\
\hline Physical fatigue & & & & $-2.275(0.010)$ & $0.395(<0.001)$ \\
\hline Reduced activity & & $-2.447(0.027)$ & & $-2.083(0.003)$ & $0.406(<0.001)$ \\
\hline Reduced motivation & & & & $-1.846(0.011)$ & $0.414(<0.001)$ \\
\hline Mental fatigue & & & & & $0.384(<0.001)$ \\
\hline
\end{tabular}

Univariate stepwise regression analysis with the following parameters: age, present use of dopamine agonists, duration of dopamine agonist use, reproductive status, and total anxiety/depression score. Data are shown as the standardized $\beta$ of independent predictive factors. $P$ values are shown in parentheses. Reproductive status: premenopausal, regular menstrual cycle $=0$; premenopausal, amenorrhea $=1$; postmenopausal $=2$. DA, dopamine agonist; $\mathrm{N}$, no; $\mathrm{Y}$, yes; yr, year. 
patients with other pituitary diseases, including acromegaly, Cushing's disease, nonfunctioning pituitary macroadenoma, and craniopharyngioma (8-10, 16). Strikingly, perceived well-being in patients treated for acromegaly is especially decreased due to physical limitations, whereas impaired quality of life in microprolactinoma patients is due to anxiety and depression. In patients with Cushing's disease and nonfunctioning pituitary macroadenoma, the quality of life is determined by fatigue and physical ability. Although quality of life is most severely impaired in patients during long-term follow-up after treatment for acromegaly (worse score on all 21 subscales of the four questionnaires when compared with own controls) (8) and Cushing's disease (worse score on 20 subscales of the same questionnaires when compared with own controls) (9), there might be disease-specific patterns in the long-term effects of pituitary diseases on quality of life parameters.

We used four health-related questionnaires to cover different dimensions of quality of life, including physical, emotional, mental, and social aspects, which were validated for Western European subjects. These healthrelated questionnaires have not been developed to assess quality of life specifically in patients with microprolactinoma, although the NHP and SF-36 are increasingly used in patients with pituitary disease $(7,25)$. However, a disease-specific questionnaire such as developed for acromegaly (Acromegaly-Quality of Life) by Webb et al. is not available for prolactinoma (26). A disease-specific questionnaire for prolactinoma should also include questions related to gonadal and sexual function, as hyperprolactinemia results in secondary hypogonadism and negatively influences libido, mood, and interest. In our study, $25 \%$ of the premenopausal women had amenorrhea, denoting hypogonadism. It is likely that well-being is influenced by hypogonadism in these patients. It is difficult to access whether hyperprolactinemia and/or hypogonadism cause(s) decreased quality of life in these female patients treated for microprolactinoma with dopamine agonists.

The interpretation of the underlying mechanisms of the association between pituitary adenoma and impaired quality of life is not completely answered. Unlike the patients studied in previous quality of life studies treated for acromegaly, Cushing's disease, or nonfunctioning macroadenoma, patients treated for microprolactinoma did not receive surgery and/or radiotherapy. In addition, they did not have pituitary deficiencies that could influence the quality of life parameters. The results of the regression analysis suggest a compromised quality of life due to the influence of anxiety and depression in these female patients treated for microprolactinoma. Despite withdrawal of dopamine agonists or normalization of prolactin levels, patients experience severe anxiety and depressive emotions, which can certainly influence experienced wellbeing. Treatment with dopamine agonists can elicit sideeffects that could affect perceived well-being. However, neither the kind of dopamine agonist therapy, nor the present use of dopamine agonists, nor duration of dopamine agonist use showed to have influence on experienced well-being. Several points of view are possible to understand the relationship of prolactinoma and emotions, including the effects of hyperprolactinemia on the central nervous system, effects of hyperprolactinemia on peripheral tissues, and emotional changes associated with the knowledge of the presence of a pituitary tumor. There is an increasing interest in the interaction between the limbic-hypothalamic system and hormone release/action. In the pathophysiology of depression, the neurotransmitter serotonin is very important (27). There is evidence for a stimulatory role of serotonin in prolactin release in humans (27). Pharmacologic agents that increase synaptic availability (e.g., re-uptake inhibitors) of serotonin, used in the treatment of depression, have been found to increase prolactin concentrations. Conversely, the density and activity of dopamine receptors are modulated by prolactin and exerts an influence on dopamine transmission in the brain (28). The turnover of dopamine is inhibited by hyperprolactinemia in some central nervous structures and stimulated in others (28). Moreover, hyperprolactinemia has been documented to induce neural changes that may, ultimately, translate into changes in behavior, emotions, and feelings (28). In addition to these putative effects of hyperprolactinemia, quality of life parameters may also be affected by the effects of dopamine agonist therapy on the brain. There are five dopamine receptor subtypes, each with specific neuroanatomical localization and function. Dopamine agonist for the D2 receptor, such as cabergoline, bromocriptine, and quinagolide, are used to treat disorders such as Parkinson disease, drug abuse, and restless legs (29). Dopamine D2 antagonist have been successfully applied in the treatment of schizophrenia, mania, and other psychiatric disorders. Therefore, it is possible that the impaired quality of life in patients with microprolactinoma is related to the effects of previous exposure to hyperprolactinemia and/or the effects of dopamine agonist therapy.

In conclusion, female patients treated for microprolactinoma have impaired self-reported quality of life due to emotional disturbances and fatigue with increased anxiety and depressive feelings. Therefore, treatment and follow-up should not only focus on the biochemical response and regain of menstrual cycle, but also on persistent psychological impairment. Failure to recognize this association between microprolactinoma and persistently impaired perceived well-being may adversely affect patient-doctor relationships (30).

\section{References}

1 Ciccarelli A, Daly AF \& Beckers A. The epidemiology of prolactinomas. Pituitary 20058 3-6.

2 Daly AF, Rixhon M, Adam C, Dempegioti A, Tichomirowa MA \& Beckers A. High prevalence of pituitary adenomas: a crosssectional study in the province of Liege, Belgium. Journal of Clinical Endocrinology and Metabolism 200691 4769-4775. 
3 Colao A \& Lombardi G. Growth-hormone and prolactin excess. Lancet 1998352 1455-1461.

4 Gillam MP, Molitch ME, Lombardi G \& Colao A. Advances in the treatment of prolactinomas. Endocrine Reviews 200627 485-534.

5 Biswas M, Smith J, Jadon D, McEwan P, Rees DA, Evans LM, Scanlon MF \& Davies JS. Long-term remission following withdrawal of dopamine agonist therapy in subjects with microprolactinomas. Clinical Endocrinology 200563 26-31.

6 Colao A, Di Sarno A, Cappabianca P, Di Somma C, Pivonello R \& Lombardi G. Withdrawal of long-term cabergoline therapy for tumoral and nontumoral hyperprolactinemia. New England Journal of Medicine $20033492023-2033$.

7 Johnson MD, Woodburn CJ \& Vance ML. Quality of life in patients with a pituitary adenoma. Pituitary 20036 81-87.

8 Biermasz NR, van Thiel SW, Pereira AM, Hoftijzer HC, van Hemert AM, Smit JW, Romijn JA \& Roelfsema F. Decreased quality of life in patients with acromegaly despite long-term cure of growth hormone excess. Journal of Clinical Endocrinology and Metabolism 200489 5369-5376.

9 Van Aken MO, Pereira AM, Biermasz NR, van Thiel SW, Hoftijzer HC, Smit JW, Roelfsema F, Lamberts SW \& Romijn JA. Quality of life in patients after long-term biochemical cure of Cushing's disease. Journal of Clinical Endocrinology and Metabolism $2005903279-3286$.

10 Dekkers OM, van der Klaauw AA, Pereira AM, Biermasz NR, Honkoop PJ, Roelfsema F, Smit JW \& Romijn JA. Quality of life is decreased after treatment for nonfunctioning pituitary macroadenoma. Journal of Clinical Endocrinology and Metabolism 200691 3364-3369.

11 Kauppinen-Makelin R, Sane T, Sintonen H, Markkanen H, Valimaki MJ, Loyttyniemi E, Niskanen L, Reunanen A \& Stenman UH. Quality of life in treated patients with acromegaly. Journal of Clinical Endocrinology and Metabolism $2006 \mathbf{9 1}$ 3891-3896.

12 Rowles SV, Prieto L, Badia X, Shalet SM, Webb SM \& Trainer PJ. Quality of life (QOL) in patients with acromegaly is severely impaired: use of a novel measure of QOL: acromegaly quality of life questionnaire. Journal of Clinical Endocrinology and Metabolism $2005903337-3341$.

13 Lindsay JR, Nansel T, Baid S, Gumowski J \& Nieman LK. Long-term impaired quality of life in Cushing's syndrome despite initial improvement after surgical remission. Journal of Clinical Endocrinology and Metabolism 200691 447-453.

14 Sonino N, Bonnini S, Fallo F, Boscaro M \& Fava GA. Personality characteristics and quality of life in patients treated for Cushing's syndrome. Clinical Endocrinology 200664 314-318.

15 Romijn JA, Smit JW \& Lamberts SW. Intrinsic imperfections of endocrine replacement therapy. European Journal of Endocrinology 2003149 91-97.

16 Dekkers OM, Biermasz NR, Smit JW, Groot LE, Roelfsema F, Romijn JA \& Pereira AM. Quality of life in treated adult craniopharyngioma patients. European Journal of Endocrinology $2006154483-489$.
17 Eustatia-Rutten CF, Corssmit EP, Pereira AM, Frolich M, Bax JJ, Romijn JA \& Smit JW. Quality of life in longterm exogenous subclinical hyperthyroidism and the effects of restoration of euthyroidism, a randomized controlled trial. Clinical Endocrinology $200664284-291$.

18 Ware JE, Jr \& Sherbourne CD. The MOS 36-item short-form health survey (SF-36). I. Conceptual framework and item selection. Medical Care 199230 473-483.

19 Brazier JE, Harper R, Jones NM, O'Cathain A, Thomas KJ, Usherwood T \& Westlake L. Validating the SF-36 health survey questionnaire: new outcome measure for primary care. BMJ 1992 305 160-164.

20 Van der Zee KI \& Sanderman R. Het meten van de algemene gezondheidstoestand met de RAND-36, een handleiding Noordelijk Centrum Voor Gezondheidsvraagstukken, pp 1-28. Groningen, The Netherlands: Rijks Universiteit Groningen, 1992.

21 Hunt SM, McKenna SP, McEwen J, Backett EM, Williams J \& Papp E. A quantitative approach to perceived health status: a validation study. Journal of Epidemiology and Community Health $198034281-286$.

22 Hunt SM \& McEwen J. The development of a subjective health indicator. Sociology of Health and Illness 19802 231-246.

23 Smets EM, Garssen B, Bonke B \& De Haes JC. The multidimensional fatigue inventory (MFI) psychometric qualities of an instrument to assess fatigue. Journal of Psychosomatic Research $199539315-325$.

24 Zigmond AS \& Snaith RP. The hospital anxiety and depression scale. Acta Psychiatrica Scandinavica 198367 361-370.

25 McMillan CV, Bradley C, Gibney J, Russell-Jones DL \& Sonksen PH. Evaluation of two health status measures in adults with growth hormone deficiency. Clinical Endocrinology 200358 436-445.

26 Webb SM, Prieto L, Badia X, Albareda M, Catala M, Gaztambide S, Lucas T, Paramo C, Pico A, Lucas A, Halperin I, Obiols G \& Astorga R. Acromegaly Quality of Life Questionnaire (ACROQOL) a new health-related quality of life questionnaire for patients with acromegaly: development and psychometric properties. Clinical Endocrinology 200257 251-258.

27 Nicholas L, Dawkins K \& Golden RN. Psychoneuroendocrinology of depression. Prolactin. Psychiatric Clinics of North America 1998 21 341-358.

28 Sobrinho LG. Emotional aspects of hyperprolactinemia. Psychotherapy and Psychosomatics 199867 133-139.

29 Zhang A, Neumeyer JL \& Baldessarini RJ. Recent progress in development of dopamine receptor subtype-selective agents: potential therapeutics for neurological and psychiatric disorders. Chemical Reviews $2007 \mathbf{1 0 7} 274-302$.

30 Jackson JL, Kroenke K \& Chamberlin J. Effects of physician awareness of symptom-related expectations and mental disorders. A controlled trial. Archives of Family Medicine 19998 135-142.

Received 19 April 2007

Accepted 23 May 2007 